\section{CHEST
CLINIC}

Department of Pulmonary Medicine, All India Institute of Medical Sciences, Bhubaneswar, Odisha, India

\section{Correspondence to}

Manoj Kumar Panigrahi, Department of Pulmonary Medicine, All India Institute of Medical Sciences, Bhubaneswar - 751019, Odisha, India; manoj_ kp99@rediffmail.com

Received 23 April 2017 Revised 15 September 2017 Accepted 25 September 2017 Published Online First 13 October 2017

\title{
Unusual cause of opaque hemithorax
}

\author{
Satyajeet Sahoo, Manoj Kumar Panigrahi, Sourin Bhuniya, Prasanta Raghab Mohapatra
}

A 40-year-old male presented with progressive breathlessness and hoarseness of voice. He denied any history of chest pain, cough, haemoptysis, palpitations or paroxysmal nocturnal dyspnoea. He was a smoker with 10 pack-year of smoking. His medical illness includes longstanding hypertension of 10 years duration controlled with oral amlodipine and atenolol. Previous echocardiography showed dilated and hypertrophied left ventricle with severe systolic dysfunction. At presentation, he was fully conscious with vitals as follows: heart rate 124/ min, respiratory rate $34 / \mathrm{min}$ and blood pressure 110/70 mm Hg. All peripheral pulses were well felt. Chest examination revealed diminished movement of left hemithorax, reduced vocal resonance and breath sound with shift of mediastinum to right. Posteroanterior chest radiograph showed an opaque left hemithorax and a large retrocardiac opacity (arrows) with contralateral shift of mediastinum (figure 1A). Ultrasonography of thorax showed a large mixed echogenic lesion with underlying collapse-consolidation of left lung and the absence of free fluid in pleural space. CT angiogram demonstrated a Stanford type $\mathrm{B}$ aortic dissection extending from proximal arch to the aortic bifurcation (figures $1 \mathrm{C}$ and 2 ). The dilated aorta caused compression of left main bronchus resulting in complete collapse of left lung (figure 1B). The patient was referred for immediate cardiothoracic intervention.

The usual causes of opaque hemithorax include massive pleural effusion, large pulmonary mass, destroyed lung, consolidation, collapse and agenesis of lung. Unilateral lung collapse occurs due to bronchial obstruction by an endobronchial lesion,

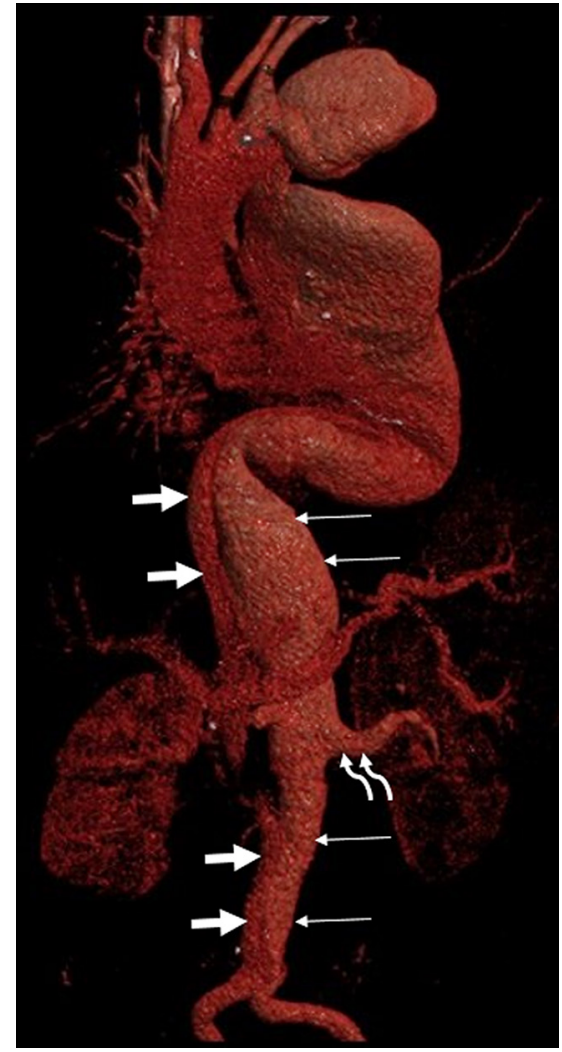

Figure 2 Volume-rendered anteroposterior coronal CT image delineating the false (thin arrows) and true (thick arrows) lumens of aorta. The left renal artery is seen coming off from the false lumen (curved arrows).

mucous plugging, foreign body aspiration and extrinsic compression from a tumour or lymph node. Descending aortic aneurysm presenting as

\section{SLinked}

- http://dx.doi.org/10.1136/ thoraxjnl-2017-210784

Check for updates

To cite: Sahoo S,

Panigrahi MK, Bhuniya S,

et al. Thorax

2018:73:395-396.
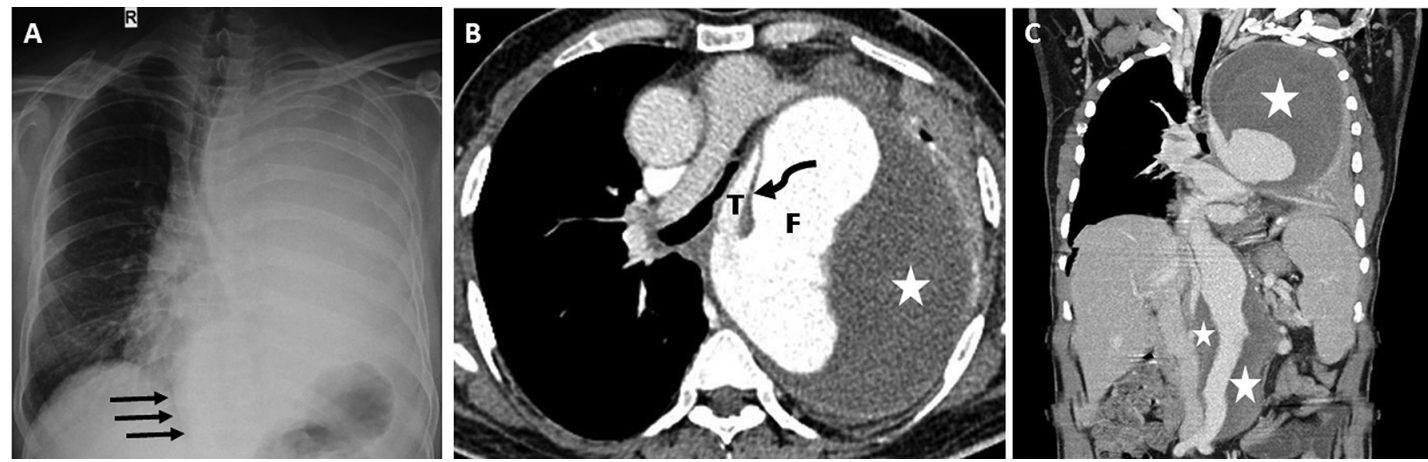

Figure 1 (A) Posteroanterior chest radiograph showing homogenous opacity occupying whole of left hemithorax and an apparent retrocardiac mass (arrows) with mediastinal shift. (B) Axial CT aortogram image (mediastinal window) at the subcarinal level showing a Stanford type B dissection with an intimal flap (curved arrow) demarcating the true (T) and false (F) lumens along with a large peripheral thrombus in situ (star) compressing the left main bronchus and pulmonary artery. (C) Coronal section image showing dissection of aorta up to the bifurcation but not extending to iliac arteries with large thrombus (stars) surrounding the thoracic and abdominal aorta. 
left lung collapse is rarely reported. ${ }^{1}$ Aortic aneurysm usually manifests as a mediastinal widening on chest radiograph that may be associated with a pleural effusion more commonly on the left side. Compression of adjacent structures by the expanding dissection and aneurysmal dilatation of aorta can lead to respiratory distress, dysphagia, superior vena cava obstruction, Horner's syndrome and hoarseness of voice (Ortner's syndrome). Aortic dissection has been classified into Stanford type A involving the ascending aorta and Stanford type B that does not involve the ascending aorta. The diagnosis is usually established by non-invasive techniques like echocardiography, CT scan and MRI. ${ }^{2}$ ECG-gated CT is considered the gold standard imaging modality in acute scenario. In view of smoking history, hoarseness of voice and unilateral opaque hemithorax a neoplastic pathology was

\section{Learning points}

- Aortic dissection can rarely present as Ortner's syndrome and unilateral opaque hemithorax.

- Thoracic ultrasonography should be routinely performed in cases of unilateral opaque hemithorax before attempting thoracentesis to avoid catastrophe like aortic puncture. initially considered in our case; however, thoracic ultrasonography and CT scan helped in timely diagnosis of aortic dissection, thereby preventing an inadvertent puncture of aorta.

Contributors SS is responsible for planning, conception of idea, collection of images and writing of initial draft. MKP is equally responsible for the conception, design and writing of the manuscript. SB helped in the writing of the manuscript, radiological description, legends for images and involved in the design of the manuscript. PRM has contributed to the writing and critically reviewed the manuscript to its final shape before submission to the journal. All authors were involved in the active management of the patient and have reviewed the final manuscript before submission to the journal. We hereby declare MKP as the guarantor for the overall content of the manuscript.

Competing interests None declared.

Patient consent Obtained.

Provenance and peer review Not commissioned; externally peer reviewed.

(C) Article author(s) (or their employer(s) unless otherwise stated in the text of the article) 2018. All rights reserved. No commercial use is permitted unless otherwise expressly granted.

\section{REFERENCES}

1 Bharty S, Prakash B, Agarwal M, et al. Opaque hemithorax: re-visiting the causes. Int J Pulm Med 2013;14:1.

2 Creager MA, Loscalzo J. Diseases of the aorta. In: Longo LD, Kasper DL, Jameson JL, eds. Harrison's principles of internal medicine. 18th edn, II. New York: Mc Graw Hill Company, 1998:2061-4. 